\title{
IMPROVEMENTS TO TURING'S METHOD II
}

\author{
TIM TRUDGIAN
}

\begin{abstract}
Turing's method uses explicit bounds on $\left|\int_{t_{1}}^{t_{2}} S(t) d t\right|$, where $\pi S(t)$ is the argument of the Riemann zeta-function. This article improves the bound on $\left|\int_{t_{1}}^{t_{2}} S(t) d t\right|$ given in [8].
\end{abstract}

1. Introduction. Let $\zeta(s)$ be the Riemann zeta-function, and let $N(T)$ denote the number of zeroes of $\zeta(s)$ with $0<\Re(s)<1$ and $0<\Im(s)<T$. One seeks to calculate $N(T)$ as follows.

First, one finds zeroes by locating sign changes of a real-valued function the zeroes of which agree with the non-trivial zeroes of the zeta-function. This gives one a lower bound on the number of zeroes of $\zeta(s)$ with $0<\Im(s)<T$.

To check whether this initial analysis has omitted some zeroes one employs Turing's method. This was first annunciated by Turing [11] in 1953 and has been used extensively since then. Recently, another method has been deployed by Büthe [2].

To apply Turing's method, one needs good explicit bounds on

$$
\left|\int_{t_{1}}^{t_{2}} S(t) d t\right|
$$

for $t_{2}>t_{1}>0$, where $\pi S(t)$ is defined to be the argument of $\zeta\left(\frac{1}{2}+i t\right)$. For a complete definition and a brief history of the problem, see [8, Section 1] and [4, Chapter 7].

This article improves [8] and contains frequent references to the results therein. The main result is

2010 AMS Mathematics subject classification. Primary 11M06.

Keywords and phrases. Turing's method, Riemann zeta-function, zeta zeroes.

Received by the editors on March 29, 2014. 


\section{Theorem 1.1.}

$$
\left|\int_{t_{1}}^{t_{2}} S(t) d t\right| \leq 1.698+0.183 \log \log t_{2}+0.049 \log t_{2},
$$

for $t_{2}>t_{1}>10^{5}$. If the right-side of (1.1) is replaced by $a+b \log \log t_{2}+$ $c \log t_{2}$, one may use Table 1 in Section 3 for more specific values of $a, b$ and $c$.

In [8], the main result followed from Lemma 2.8 and Lemma 2.11 which concerned, respectively, obtaining an upper and a lower bound for $\Re \log \zeta(s)$ for $\Re(s) \geq \frac{1}{2}$. This article refines only the upper bound. Theorem 1.1 improves on Theorem 2.2 in [8] for all $t_{2} \geq 10^{5}$.

The idea in this article is to use more sophisticated estimates on $\zeta(\sigma+i t)$ for $\frac{1}{2} \leq \sigma \leq 1$; these estimates have been given in $[7,10]$. A bound on $|\zeta(s)|$ is given in Section 2, a proof of Theorem 1 is given in Section 3, and some concluding remarks are provided in Section 4.

2. Bounding $|\zeta(\sigma+i t)|$ across the strip $\frac{1}{2} \leq \sigma \leq 1+\delta$. Using the inequality $\log (1+x) \leq x$, it is easy to see that

$$
\log \left|Q_{0}+\sigma+i t\right|-\log t \leq \frac{1}{2}\left(\frac{\sigma_{1}+Q_{0}}{t_{0}}\right)^{2},
$$

for $\sigma \leq \sigma_{1}$ and $t \geq t_{0}$ and any $Q_{0} \geq 0$. With the trivial observations $\log \left|Q_{0}+\sigma+i t\right| \geq \log t$ and $\left|\arg \left(Q_{0}+\sigma+i t\right)\right| \leq \frac{\pi}{2}$ at hand, we may apply (2.1) to see that

$$
\left|\log \left(Q_{0}+\sigma+i t\right)\right| \leq\left(1+a_{0}\right) \log t, \quad\left(\sigma \leq \sigma_{1}\right),
$$

where

$$
a_{0}=a_{0}\left(\sigma_{1}\right)=\frac{\sigma_{1}+Q_{0}}{2 t_{0}^{2} \log t_{0}}+\frac{\pi}{2 \log t_{0}}+\frac{\pi\left(\sigma_{1}+Q_{0}\right)^{2}}{4 t_{0} \log ^{2} t_{0}} .
$$

Suppose that

$$
\begin{aligned}
\left|\zeta\left(\frac{1}{2}+i t\right)\right| & \leq k_{1} t^{k_{2}}(\log t)^{k_{3}}, & & \left(t \geq t_{1}\right), \\
|\zeta(1+i t)| & \leq k_{4} \log t^{k_{5}}, & & \left(t \geq t_{2}\right) .
\end{aligned}
$$

Consider the function $h(s)=(s-1) \zeta(s)$, which is entire. Once we are able to exhibit bounds for $|h(s)|$ using the information in (2.3), we 
can apply a version of the Phragmén-Lindelöf principle to bound $|\zeta(s)|$. Using [9, Lemma 3] and (2.1) and (2.2), we may prove

Lemma 2.1. Let $h(s)=(s-1) \zeta(s)$, and let $\delta$ be a positive real number. Furthermore, let $Q_{0} \geq 0$ be a number for which

$$
\begin{aligned}
\left|h\left(\frac{1}{2}+i t\right)\right| & \leq k_{1}\left|Q_{0}+\frac{1}{2}+i t\right|^{k_{2}+1}\left(\log \left|Q_{0}+\frac{1}{2}+i t\right|\right)^{k_{3}} \\
\mid h(1+i t) & \leq k_{4}\left|Q_{0}+1+i t\right|\left(\log \left|Q_{0}+1+i t\right|\right)^{k_{5}} \\
|h(1+\delta+i t)| & \leq \zeta(1+\delta)\left|Q_{0}+1+\delta+i t\right|,
\end{aligned}
$$

for all $t$. Then, for $\sigma \in\left[\frac{1}{2}, 1\right]$ and $t \geq t_{0}$,

$$
|\zeta(s)| \leq \alpha_{1} k_{1}^{2(1-\sigma)} k_{4}^{2(\sigma-1 / 2)} t^{2 k_{2}(1-\sigma)}(\log t)^{2\left(k_{3}(1-\sigma)+k_{5}(\sigma-1 / 2)\right)},
$$

where

$$
\alpha_{1}=\left(1+a_{1}\left(1+\delta, Q_{0}, t_{0}\right)\right)^{k_{2}+1}\left(1+a_{0}\left(1+\delta, Q_{0}, t_{0}\right)\right)^{k_{3}+k_{5}},
$$

and

$$
\begin{aligned}
& a_{0}\left(\sigma, Q_{0}, t\right)=\frac{\sigma+Q_{0}}{2 t^{2} \log t}+\frac{\pi}{2 \log t}+\frac{\pi\left(\sigma+Q_{0}\right)^{2}}{4 t \log ^{2} t}, \\
& a_{1}\left(\sigma, Q_{0}, t\right)=\frac{\sigma+Q_{0}}{t}
\end{aligned}
$$

whereas for $\sigma \in[1,1+\delta]$ and $t \geq t_{0}$,

$$
|\zeta(s)| \leq \alpha_{2} k_{4}^{(1+\delta-\sigma) / \delta} \zeta(1+\delta)^{(\sigma-1) / \delta}(\log t)^{k_{5}(1+\delta-\sigma) / \delta},
$$

where

$$
\alpha_{2}=\left(1+a_{1}\left(1+\delta, Q_{0}, t_{0}\right)\right)\left(1+a_{0}\left(1+\delta, Q_{0}, t_{0}\right)\right)^{k_{5}} .
$$

Finally, for all $\sigma \in\left[\frac{1}{2}, 1+\delta\right]$ and $t \geq t_{0}$, we have

$$
|\zeta(s)| \leq\left(1+a_{1}\left(1+\delta, Q_{0}\right)\right)^{k_{2}+1}\left(1+a_{0}\left(1+\delta, Q_{0}\right)\right)^{k_{3}+k_{5}} k_{1} t^{k_{2}}(\log t)^{k_{3}},
$$

provided that

$$
t^{k_{2}}(\log t)^{k_{3}-k_{5}} \geq \frac{k_{4}}{k_{1}}, \quad t \geq \exp \left\{\left(\frac{\zeta(1+\delta)}{k_{4}}\right)^{1 / k_{5}}\right\}
$$


Proof. In applying Lemma 3 of [9] to $h(s)$, we need to relate $\left|Q_{0}+s\right|$ and $|s-1|$ to $t$. We simply note that

$$
\begin{aligned}
\left|\frac{Q_{0}+s}{s-1}\right| & \leq \frac{\left|Q_{0}+s\right|}{t} \leq 1+\frac{\sigma+Q_{0}}{t_{0}} \\
& =1+a_{1}\left(\sigma, Q_{0}\right) \leq 1+a_{1}\left(1+\delta, Q_{0}\right),
\end{aligned}
$$

in both regions $\sigma \in\left[\frac{1}{2}, 1\right]$ and $\sigma \in[1,1+\delta]$. Since $a_{0}$ and $a_{1}$ are small for any respectable value of $t_{0}$, we throw away some information in the exponents of $1+a_{1}$ and $1+a_{0}$. For example, in proving (2.4), we arrive at

$$
\left(1+a_{1}\right)^{2 k_{2}(1-\sigma)+1}\left(1+a_{0}\right)^{2 k_{3}(1-\sigma)+2 k_{5}(\sigma-1 / 2)} .
$$

Rather than retain this dependence on $\sigma$ in the exponents, we simply bound $1-\sigma$ and $\sigma-\frac{1}{2}$ by $\frac{1}{2}$. A similar procedure is applied to prove $(2.5)$.

To prove the bound in the region $\sigma \in\left[\frac{1}{2}, 1+\delta\right]$, we note that the bounds in (2.4) and (2.5) are decreasing in $\sigma$ if the inequalities in (2.6) are met. Finally, the bound in (2.4), evaluated at $\sigma=\frac{1}{2}$, exceeds the bound in (2.5), evaluated at $\sigma=1$. This completes the lemma.

It is worth recording the values of $k_{1}, \ldots, k_{5}$, which we do in

Corollary 2.2. For $\sigma \in\left[\frac{1}{2}, 1+\delta\right]$ and $t \geq t_{0}$, we have

$$
|\zeta(s)| \leq 0.732\left(1+a_{1}\left(1+\delta, 5, t_{0}\right)\right)^{7 / 6}\left(1+a_{0}\left(1+\delta, 5, t_{0}\right)\right)^{2} t^{1 / 6} \log t,
$$

provided that

$$
t \geq \max \{1.16, \exp [4 \zeta(1+\delta) / 3]\}
$$

Proof. In [10], it was shown that $|\zeta(1+i t)| \leq \frac{3}{4} \log t$ for $t \geq 3$. In [7], it was shown that $\left|\zeta\left(\frac{1}{2}+i t\right)\right| \leq 0.732|4.678+i t|^{1 / 6} \log |4.678+i t|$ for all $t$. One may therefore choose

$$
\left(k_{1}, k_{2}, k_{3}, k_{4}, k_{5}, Q_{0}\right)=\left(0.732, \frac{1}{6}, 1, \frac{3}{4}, 1,5\right)
$$

in Lemma 2.1, which proves the corollary.

Although we shall use (2.7) in our computation we proceed with the variables $\left(k_{1}, \ldots, Q_{0}\right)$ as parameters. We remark that, although 
Corollary 2.2 is not used in this article, it is derived at very little additional cost and should prove useful for related problems.

3. Proof of Theorem 1.1. We are now able to proceed to the proof of Theorem 1.1. We need to give an upper bound for

$$
I=\Re \int_{1 / 2+i t}^{\infty+i t} \log \zeta(s) d s .
$$

To that end, we shall write

$$
I \leq \int_{1 / 2+i t}^{1+i t} \log |\zeta(s)| d s+\int_{1+i t}^{1+\delta+i t} \log |\zeta(s)| d s+\int_{1+\delta}^{\infty} \log |\zeta(\sigma)| d \sigma,
$$

and apply (2.4) to the first integral in (3.1) and (2.5) to the second. This gives us

Lemma 3.1. For $t \geq t_{0}$, we have

$$
I \leq A_{1}+B_{1} \log \log t+C_{1} \log t
$$

where

$$
\begin{aligned}
A_{1}= & \int_{1+\delta}^{\infty} \log |\zeta(\sigma)| d \sigma+\left(\frac{k_{2}}{4}+\frac{1}{2}+\delta\right) \log \left(1+a_{1}\left(1+\delta, Q_{0}, t_{0}\right)\right) \\
& +\left(\frac{k_{3}}{4}+\frac{k_{5}}{4}+\frac{k_{5} \delta}{2}\right) \log \left(1+a_{0}\left(1+\delta, Q_{0}, t_{0}\right)\right) \\
& +\left(\frac{1}{4}+\frac{\delta}{2}\right) \log k_{4}+\frac{1}{4} \log k_{1}+\frac{\delta}{2} \log \zeta(1+\delta),
\end{aligned}
$$

and

$$
B_{1}=\left(\frac{k_{3}+k_{5}}{4}+\frac{\delta k_{5}}{2}\right), \quad C_{1}=\frac{k_{2}}{4} .
$$

The term corresponding to $C_{1}$ in [8, Lemma 2.8] is $k_{2} / 4+\delta k_{2} / 2$. Since we are not permitted to take $\delta$ too small lest the integral in $A_{1}$ become too large, this represents a considerable qualitative saving. This is due entirely to estimating $|\zeta(s)|$, not in one go over $\sigma \in\left[\frac{1}{2}, 1+\delta\right]$ as in [8], but by using Lemma 2.1. We combine Lemma 3.1 with [8, Lemma 2.11] to obtain 
Theorem 3.2. For $t_{2} \geq t_{1} \geq 10^{5}$,

$$
\left|\int_{t_{1}}^{t_{2}} S(t) d t\right| \leq a+b \log \log t_{2}+c \log t_{2}
$$

where

$$
\begin{aligned}
\pi a= & \int_{1+\delta}^{\infty} \log |\zeta(\sigma)| d \sigma+\left(\frac{k_{2}}{4}+\frac{1}{2}+\delta\right) \log \left(1+a_{1}\left(1+\delta, Q_{0}, 10^{5}\right)\right) \\
& +\frac{1}{4} \log k_{1}+\left(\frac{k_{3}}{4}+\frac{k_{5}}{4}+\frac{k_{5} \delta}{2}\right) \log \left(1+a_{0}\left(1+\delta, Q_{0}, 10^{5}\right)\right) \\
& +\left(\frac{1}{4}+\frac{\delta}{2}\right) \log k_{4}+\frac{\delta}{2} \log \zeta(1+\delta)+\frac{d^{2}}{2} \log \pi+3 \times 10^{-4} \\
& +d^{2}(\log 4)\left\{-\frac{\zeta^{\prime}(1 / 2+d)}{\zeta(1 / 2+d)}-\frac{1}{2} \log 2 \pi+\frac{1}{4}\right\}-\frac{1}{2} \int_{1+2 d}^{\infty} \log \zeta(\sigma) d \sigma \\
& +\int_{1 / 2+d}^{\infty} \log \zeta(\sigma) d \sigma-\frac{1}{2} \int_{1+2 d}^{1+4 d} \log \zeta(\sigma) d \sigma+\int_{1 / 2+d}^{1 / 2+2 d} \log \zeta(\sigma) d \sigma,
\end{aligned}
$$

and

$$
\pi b=\left(\frac{k_{3}+k_{5}}{4}+\frac{\delta k_{5}}{2}\right), \quad \pi c=\frac{k_{2}}{4}+\frac{d^{2}}{2}(\log 4-1) .
$$

3.1. Computation. Before we commence an analysis of the coefficients appearing in Theorem 3.2 we make the following observation. One may replace the values of $\left(k_{1}, k_{2}, k_{3}\right)$ in $(2.7)$ by

$$
\left(k_{1}, k_{2}, k_{3}\right)=\left(\frac{4}{(2 \pi)^{1 / 4}}, \frac{1}{4}, 0\right),
$$

which appear in [5, Lemma 2]. The values in (3.4) are obtained using the approximate functional equation of $\zeta(s)$ : the values in $(2.7)$ are obtained using exponential sums. The value $k_{2}=\frac{1}{4}$ follows from convexity theorems. We call (3.4) the convexity result and (2.7) the sub-convexity result.

As in [8, Theorem 2.12], no term in either (3.2) or (3.3) depends on both $\delta$ and $d$. We can run two one-dimensional optimizations on each of $a, b$ and $c$. In Table 1, we compare the results obtained from the convexity result $(\mathrm{C})$, the sub-convexity result $(\mathrm{SC})$, and the coefficients in [8, Theorem 2.2], when $t_{1} \approx T$. The values of $\delta, d, a, b$ and $c$ 
correspond to the sub-convexity result. We find that the sub-convexity result overtakes the convexity result when $T \geq 2.85 \times 10^{10}$, which is, just barely, beneath the height to which the Riemann hypothesis has been verified - see [6].

The values used in Theorem 1.1 are taken from the row $T=10^{10}$. It should be stressed that all of the results in this table are valid for $t_{1} \geq 10^{5}$. The stated values of $a, b$ and $c$ are those that are close to the best values obtainable by this method when $t_{1} \approx T$.

TABLE 1. Comparison of bounds for $\left|\int_{t_{1}}^{t_{2}} S(t) d t\right| \leq a+b \log \log t_{2}+c \log t_{2}$.

\begin{tabular}{ccccccccc}
\hline \hline$T$ & Theorem 2.2 & $\mathrm{C}$ & $\mathrm{SC}$ & $d$ & $\delta$ & $a$ & $b$ & $c$ \\
\hline $10^{5}$ & 2.747 & 2.629 & 2.658 & 0.883 & 0.279 & 1.457 & 0.204 & 0.062 \\
$10^{6}$ & 2.883 & 2.800 & 2.827 & 0.845 & 0.237 & 1.520 & 0.197 & 0.058 \\
$10^{7}$ & 3.018 & 2.959 & 2.982 & 0.817 & 0.206 & 1.573 & 0.192 & 0.055 \\
$10^{8}$ & 3.154 & 3.110 & 3.128 & 0.795 & 0.182 & 1.620 & 0.189 & 0.053 \\
$10^{9}$ & 3.290 & 3.255 & 3.266 & 0.777 & 0.163 & 1.661 & 0.186 & 0.051 \\
$10^{10}$ & 3.426 & 3.395 & 3.398 & 0.762 & 0.148 & 1.698 & 0.183 & 0.049 \\
$10^{11}$ & 3.562 & 3.530 & 3.526 & 0.749 & 0.135 & 1.733 & 0.181 & 0.048 \\
$10^{12}$ & 3.698 & 3.663 & 3.649 & 0.738 & 0.124 & 1.764 & 0.179 & 0.047 \\
$10^{13}$ & 3.834 & 3.792 & 3.770 & 0.729 & 0.115 & 1.792 & 0.178 & 0.046 \\
$10^{14}$ & 3.969 & 3.919 & 3.887 & 0.720 & 0.107 & 1.820 & 0.177 & 0.046 \\
$10^{15}$ & 4.105 & 4.044 & 4.002 & 0.713 & 0.100 & 1.844 & 0.176 & 0.045 \\
\hline \hline
\end{tabular}

4. Conclusion. It seems difficult to improve substantially on Theorem 1.1. Given that the improvements obtained in this paper are only modest, and since further improvements would require a lot of effort in estimating $\zeta\left(\frac{1}{2}+i t\right)$ or $\zeta(1+i t)$, it seems hopeless to try to improve this part of the argument.

One could try one's luck at reducing the term $\log 4$ that appears in both (3.2) and (3.3). This comes from [1, Lemma 4.4]. Reducing this would have a more profound influence on bounding $\left|\int_{t_{1}}^{t_{2}} S(t) d t\right|$ than better bounds for $|\zeta(s)|$.

Finally, it is worth considering [8, Theorems 3.3 and 4.3], which relate to Dirichlet $L$-functions and Dedekind zeta-functions. Both of these could be improved, in line with this article, were one in possession of explicit estimates on the lines $\sigma=\frac{1}{2}$ and $\sigma=1$. One such estimate, 
bounding $|L(1+i t, \chi)|$ for $L(s)$ a Dirichlet $L$-function, appears in [3]. It is possible that this could be used to obtain an improvement to $[\mathbf{8}$, Theorem 3.3].

Acknowledgments. I should like to thank Jan Büthe for detailed discussions on this problem.

\section{REFERENCES}

1. A.R. Booker, Artin's conjecture, Turing's method, and the Riemann hypothesis, Experiment. Math. 15 (2006), 385-407.

2. J. Büthe, A method for proving the completeness of a list of zeros of certain L-functions, Math. Comp. 84 (2015), 2413-2431.

3. A.W. Dudek, An explicit result for $|L(1+i t, \chi)|$, Funct. Approx. Comm. Math. 53 (2015), 23-29.

4. H.M. Edwards, Riemann's zeta function, Pure Appl. Math., Academic Press, New York, 1974.

5. R.S. Lehman, On the distribution of zeros of the Riemann zeta-function, Proc. Lond. Math. Soc. 3 (1970), 303-320.

6. D.J. Platt, Computing $\pi(x)$ analytically, Math. Comp. 84 (2015), 1521-1535.

7. D.J. Platt and T.S. Trudgian, An improved explicit bound on $\left|\zeta\left(\frac{1}{2}+i t\right)\right|$, J. Num. Th. 147 (2015), 842-851.

8. T.S. Trudgian, Improvements to Turing's method, Math. Comp. 80 (2011), 2259-2279.

9. An improved upper bound for the argument of the Riemann zetafunction on the critical line II, J. Num. Th. 134 (2014), 280-292.

10. , A new upper bound for $|\zeta(1+i t)|$, Bull. Aust. Math. Soc. 89 (2014), $259-264$.

11. A.M. Turing, Some calculations of the Riemann zeta-function, Proc. Lond. Math. Soc. 3 (1953), 99-117.

Mathematical Sciences Institute, Building 27 The Australian National University, Canberra ACT 0200, Australia

Email address: timothy.trudgian@anu.edu.au 